\title{
Relationship of Ethylene Production to Stress Alleviation in Seeds of Lettuce Cultivars
}

\author{
Janusz Prusinski'and Anwar A. Khan ${ }^{2}$ \\ Department of Horticultural Sciences, New York State Agricultural Experiment Station, Cornell \\ University, Geneva, NY 14456
}

Additional index words. Lactuca sativa, ethephon, 1-aminocyclopropane- 1-carboxylic acid, salt stress, osmotic stress, thermoinhibition

\begin{abstract}
Seeds (intact or slit) of lettuce (Luctuca sativa L.) cultivars with greater ability to produce ethylene germinated better under stressful conditions. Highly significant correlations were found between ethylene production and germination in $0.1 \mathrm{M} \mathrm{NaCI}$ ( - 0.49 MPa) solution at 25C $(r=0.95$, intact seeds), in - 0.3 MPa PEG solution $(r=0.86$, intact seeds; $r=0.81$, slit seeds), and in water at $32 \mathrm{C}(r=0.80$, slit seeds) or 35C $(r=0.80$, slit seeds). Slitting the seed coat increased the ethylene production and improved germination during osmotic restraint in most cultivars, particularly in 'Mesa 659' and 'Super 59'. The differing ability of cultivars to produce ethylene during stress generally corresponded with their ability to generate germination potential. Ethylene production and germination potential in untreated and ACC-treated 'Mesa 659' seeds increased upon slitting under stressful conditions. Thus, the ability of seeds to produce ethylene and to generate high germination potential under stressful conditions may be used as criteria to select stress-tolerant lettuce cultivars. Chemical names used: polyethylene glycol 8000 (PEG), 1-aminocyclopropane- 1-carboxylic acid (ACC), (2-chlorethyl) phosphoric acid (ethephon).
\end{abstract}

Inhibition of germination of lettuce seeds by high temperature (Abeles, 1986; Braun and Khan, 1976; Rao et al., 1975; Sharples, 1973), salinity (Braun and Khan, 1976; Khan and Huang, 1988; Negro and Smith, 1978), or an osmoticum (Abeles, 1986; Braun and Khan, 1976; Negro and Smith, 1978) can be reduced or prevented by ethylene. Both ethylene synthesis and ethylene action have been implicated in the alleviation of stress on lettuce seed germination (Abeles, 1986; Khan and Huang, 1988; Saini et al., 1986). In rice, ethylene production correlated well with seedling growth under saline (Khan et al., 1987) and high-temperature (Khan, 1989) conditions. Seeds and seedlings have greater sensitivity to ethylene under stress than under nonstress conditions, which may indicate a greater need of ethylene for growth processes under stress (Khan, 1989; Khan et al., 1987). The conversion of ACC into ethylene decreases as the temperature is increased from 25 to 35C (Khan and Prusinski, 1989).

Takeba and Matsubara (1979) showed that the embryo growth or germination potential of 'New York' lettuce decreased greatly with an increase in temperature and at $35 \mathrm{C}$ the potential was less than that required to remove the seed coat restraint. Preliminary studies from this laboratory show a wide variability in lettuce cultivars for germination at high temperatures.

This investigation was undertaken to: a) determine if pregermination ethylene production by seeds of lettuce cultivars was related to their capacity to germinate under stressful conditions, b) ascertain if lettuce cultivars differ in their capacity to generate germination potential, and c) determine if the seed coat reduces the conversion of ACC into ethylene and thus acts as a barrier to ethylene production and/or action in the generation of germination potential.

\footnotetext{
Received for publication 8 May 1989. This work was partially supported by a grant from the Ferry Morse Seed Co., Modesto, Calif. Gifts of (2-chloroethyl) phosphoric acid from Union Carbide Corp., Research Triangle Park, N. C., and of seeds of lettuce cultivars from Ferry Morse Seed Co., Modesto, Calif., and Harris-Moran Seed Co., Rochester, N. Y., are greatly appreciated. The cost of publishing this paper was defrayed in part by the payment of page charges. Under postal regulations, this paper therefore must be hereby marked advertisement solely to indicate this fact.

'Research Fellow on leave from Plant Cultivation Dept., Technical-Agricultural Univ., Bydgoszcz, Poland.

'To whom reprint requests should be addressed.
}

\section{Materials and Methods}

Lettuce cultivars Emperor, Super 59, Fanfare, Empress, Garnet, and Grand Rapids were obtained from Ferry-Morse Seed Company, Modesto, Calif.; and 'Mesa 659', 'Montello', and 'Ithaca' from Harris Moran Seed Co., Rochester, N.Y. All seeds were from 1988 harvest, except 'Grand Rapids', which was harvested in 1986. Seeds were kept at $\approx 7 \mathrm{C}$ and $28 \% \mathrm{RH}$ and small amounts were removed as needed.

Germination. Fifty intact or slit seeds in batches of 50 (three replicates) were soaked in $5.0-\mathrm{cm}$ petri plates lined with two layers of Whatman No. 1 filter paper and moistened with $3 \mathrm{ml}$ of water or test solution. Seeds were slit by making a longitudinal cut at the cotyledon end about one-third the length of seed. For high-temperature stress, seeds were moistened with water and incubated at $32 \pm 1 \mathrm{C}$ or $35 \pm 1 \mathrm{C}$; for salt stress, seeds were incubated at $25 \pm 1 \mathrm{C}$ in $0.1 \mathrm{M} \mathrm{NaCl}(-4.9 \mathrm{MPa}$, based on Vant'Hoff's equation), and for osmotic stress at $25 \pm 1 \mathrm{C}$ in - 0.3 MPa polyethylene glycol 8000 (PEG) solution. Germination was recorded after $16 \mathrm{hr}$ of incubation. Seeds were considered germinated when radicle protrusion (typical germination) or cotyledon protrusion [atypical germination in slit seeds (Ikuma and Thimann, 1963)] occurred.

Ethylene determination. Fifty seeds (three replicates) were soaked on filter paper for $10 \mathrm{hr}$ at $25 \mathrm{C}$ in $0.1 \mathrm{M} \mathrm{NaCl}$ or -0.3 MPa PEG solution or $10 \mathrm{hr}$ at 32 or $35 \mathrm{C}$ in water, rapidly wipedried on paper towels, transferred to 5.7-ml glass tubes, and capped with a rubber septum. Tubes were incubated for $2 \mathrm{hr}$ on their side at $25 \mathrm{C}$ in light $\left(9 \mu \mathrm{mol} \cdot \mathrm{s}^{-1} \cdot \mathrm{m}^{-2}\right)$. Ethylene content in the gas phase of the tube was determined by withdrawing a 1-ml sample with a gas-tight syringe and injecting into a Hewlett-Packard 5790 gas chromatography equipped with a flame ionization detector and a $183 \times 0.32-\mathrm{cm}$ stainless steel column containing Poropak Q. A correction was made in calculating ethylene diluted due to a vacuum created during removal of gas from the tubes.

Batches of $0.3 \mathrm{~g}$ of seeds were soaked in $6 \mathrm{ml}$ of water or $10 \mathrm{mM} \mathrm{ACC}$ for $2 \mathrm{hr}$ at $25 \mathrm{C}$ and dried by forced air at $25 \mathrm{C}$ for $2 \mathrm{hr}$. A portion of these seeds were slit as described previously. Fifty untreated or ACC-treated intact and slit seeds (two replicates) were incubated for $10 \mathrm{hr}$ in $0.1 \mathrm{M} \mathrm{NaCl}$ or $-0.3 \mathrm{MPa}$ PEG solution at $25 \mathrm{C}$ or in water at $35 \mathrm{C}$, and ethylene production 
determined after $1 \mathrm{hr}$ incubation at $25 \mathrm{C}$, as described meviously.

Germination potential studies. Fifty seeds (three replicates) were germinated in $3.0 \mathrm{ml}$ of PEG solution ranging in water potential $(\psi)$ from 0.0 to $-0.8 \mathrm{MPa}$ at $30 \mathrm{C}$ for $48 \mathrm{hr}$. To determine the effect of ACC and ethephon (ETH) on germination potential, seeds were presoaked for $2 \mathrm{hr}$ in water, $10 \mathrm{mM}$ ACC, or $10 \mathrm{mM} \mathrm{ETH}$, dried for $2 \mathrm{hr}$, and a portion of the seeds were slit. Fifty treated or untreated intact and slit seeds (two replicates) then were germinated at 32 or $35 \mathrm{C}$ in PEG solutions of varying $\psi$ for $48 \mathrm{hr}$. The PEG solutions were prepared and adjusted for temperature as described by Michel and Kaufmann (1973). The $\psi$ reducing germination to $50 \%$ was regarded as a measure of the germination potential (Takeba and Matsubara, 1979). Seed samples germinating $<50 \%$ were regarded as having zero germination potential. The embryo coverings of lettuce seed (pericarp, testa, and endosperm) have been collectively referred to as "seed coat" in this study.

\section{Results}

Large cultivar differences in the ability to germinate and to produce ethylene were found when lettuce seeds were soaked in PEG of $-0.3 \mathrm{MPa}$ (Fig. 1). The ethylene produced correlated well ( $r=0.86)$ with the capacity of seeds to germinate. 'Emperor', 'Ithaca', and 'Fanfare', with a relatively high capacity to produce ethylene, were among the good performers; 'Garnet', 'Montello', 'Super 59', and 'Mesa 659', which produced little ethylene, performed poorly. An even higher correlation $(r=0.95)$ was found between ethylene production and germination in lettuce cultivars under saline $(0.1 \mathrm{M} \mathrm{NaCl})$ conditions (Fig. 2). Again, 'Emperor', 'Ithaca', and 'Fanfare' outperformed 'Garnet', 'Montello', 'super 59', and 'Mesa 659'.

When lettuce seeds were slit at the cotyledon end before soaking them in osmotic solution of $-0.3 \mathrm{MPa}$, a marked increase

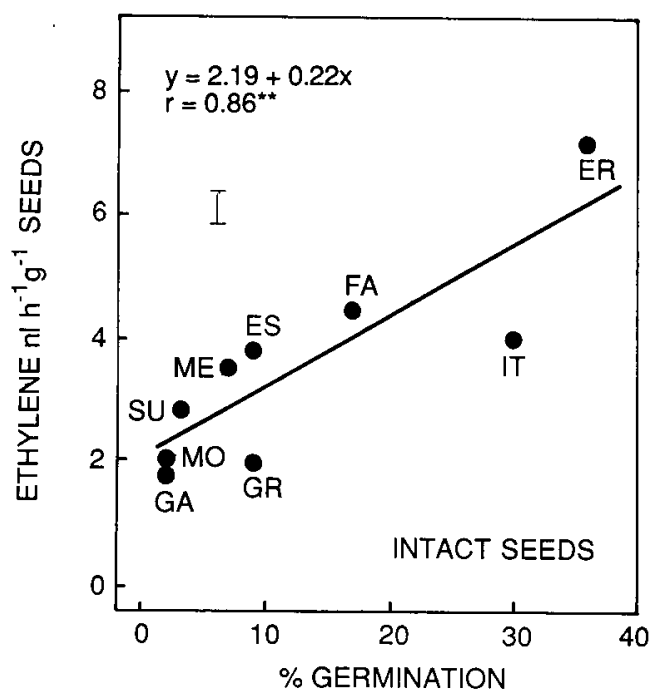

Fig. 1. Relationship between ethylene production and germination of intact seeds in nine lettuce cultivars during osmotic (PEG) stress. Germination and ethylene production were determined after a 16and 10-hr soak, respectively, in - 0.3 MPa PEG solution at 25C. Cultivar notations: ER, 'Emperor'; IT, 'Ithaca'; FA, 'Fanfare'; ES, 'Empress'; GR, 'Grand Rapids'; ME, 'Mesa 659'; SU, 'Super 59'; MO, 'Montello'; GA, 'Garnet'.

**Significance at .01 probability level. Vertical bar denotes LsD at 0.05 probability level.

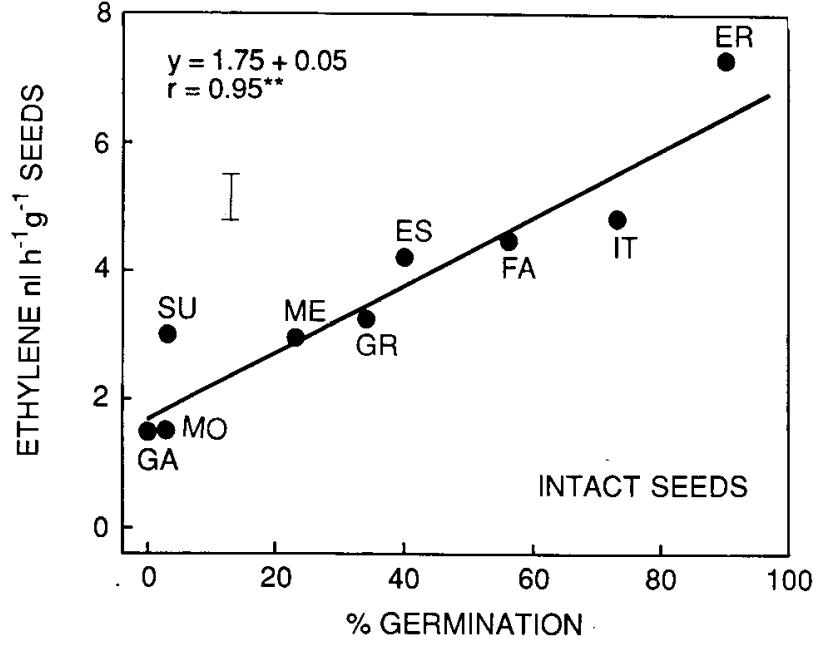

Fig. 2. Relationship between ethylene production and germination of intact seeds in lettuce cultivars during salinity $(\mathrm{NaCl})$ stress. Germination and ethylene production were determined after a 16- and 10-hr soak, respectively, in $0.1 \mathrm{M} \mathrm{NaCl}$ at $25 \mathrm{C}$. Other details as in Fig. 1.

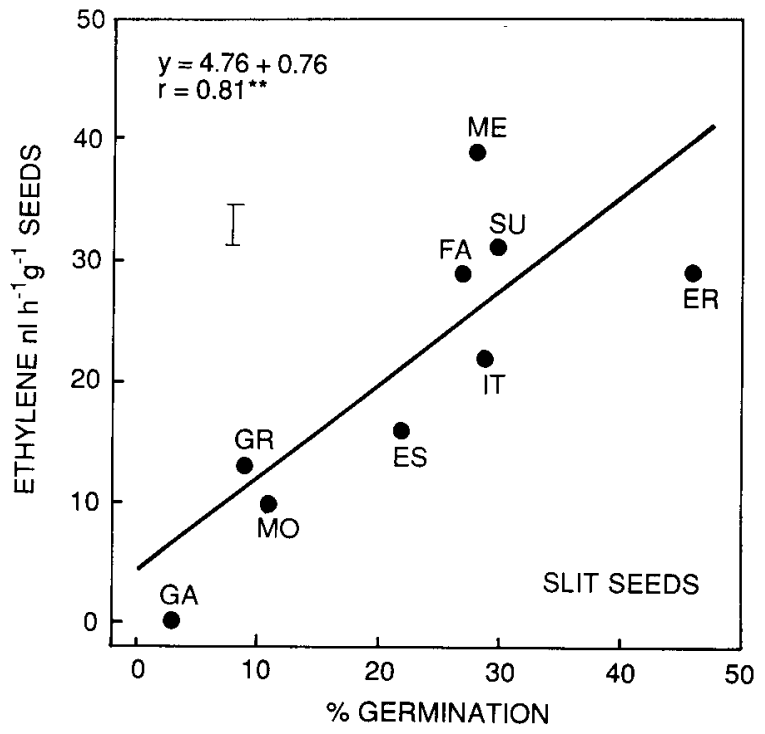

Fig. 3. Relationship between ethylene production and germination of slit seeds in lettuce cultivars during osmotic (PEG) stress. Seeds were slit by making a longitudinal cut at the cotyledon end about one-third the length of seed. Other details as in Fig. 1.

occurred in the ethylene-producing capacity of all cultivars, with the exception of 'Garnet' (compare Figs. 2 and 3). Ethylene production still correlated well $(r-=0.80)$ with the germination capacity of the seeds. More than 10-fold increases in ethylene production occurred in seeds of 'Mesa 659' (3.5 to $\left.39 \mathrm{nl} \cdot \mathrm{hr}^{-1} \cdot \mathrm{g}^{-1}\right)$ and 'Super 59' (from 2.8 to $31 \mathrm{nl} \cdot \mathrm{hr}^{-1} \cdot \mathrm{g}^{-1}$ ). Also, these cultivars responded to slitting better than others by improving their germination percentages. Slitting caused relatively smaller increases in germination percentages of 'Fanfare', 'Emperor', 'Montello', and 'Empress' and little or no increase in 'Grand Rapids', 'Garnet', and 'Ithaca'.

Ethylene produced at 32 and $35 \mathrm{C}$ during 8 to $10 \mathrm{hr}$ of soaking of intact seeds was low in most cultivars (data not shown). After slitting, ethylene production increased greatly and correlated 
well with germination, both at $32 \mathrm{C}(\mathrm{r}=0.80)$ (Fig. 4) and at 35C ( $\mathrm{r}=0.80)$ (Fig. 5). The relative performances of slit seeds of different cultivars at high temperatures were similar to their performances during osmotic stress (Figs. 3-5).

Seeds of the various cultivars differed greatly in their ability to generate germination potential at 30C (Fig. 6). The germination potential ranged from $0.57 \mathrm{MPa}$ for 'Emperor' through $0.14 \mathrm{MPa}$ for 'Super 59'. Only a few seeds of 'Montello' and none of 'Garnet' germinated at 30C. There was a similarity in the ability of cultivars to generate germination potential and their ability to produce ethylene and to alleviate salinity and osmotic stress (Figs. 1, 2, and 6).

An experiment was designed with 'Mesa 659' seeds to test if the seed coat prevented or reduced the conversion of ACC to

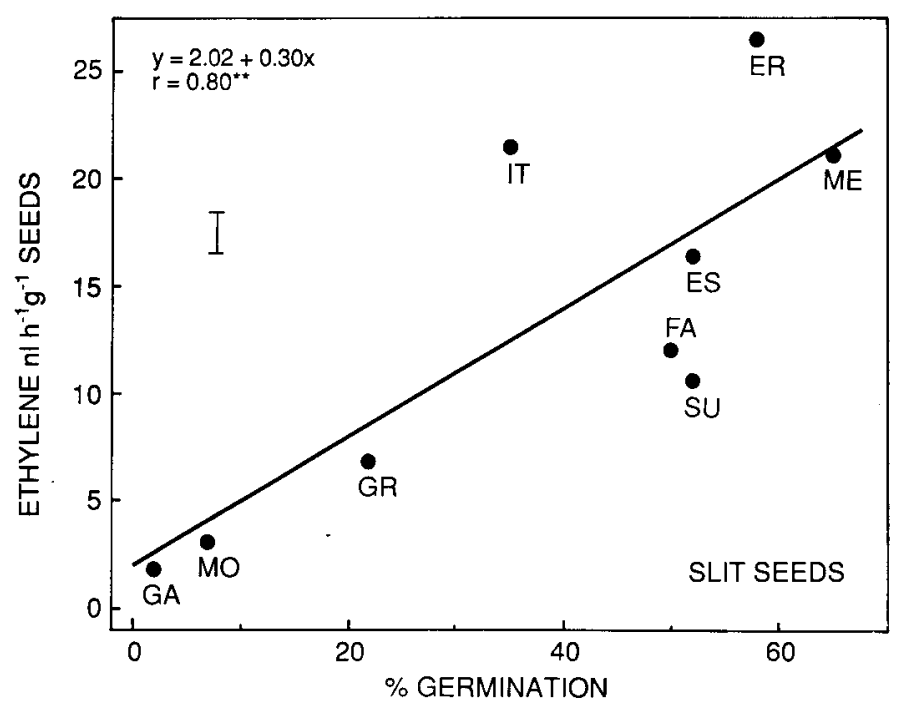

Fig. 4. Relationship between ethylene production and germination of slit seeds in lettuce cultivars at 32C. Germination and ethylene production were determined after a 16- and 10-hr soak, respectivly. Other details as in Fig. 1.

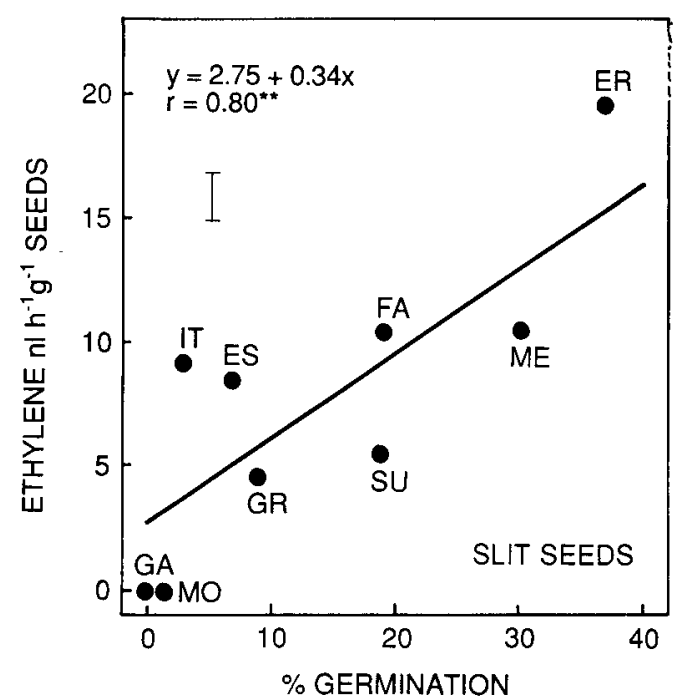

Fig. 5. Relationship between ethylene production and germination of slit seeds at 35C. Germination and ethylene production were determined after a 16- and 10-hr soak, respectively. Other details as in Fig. 1.

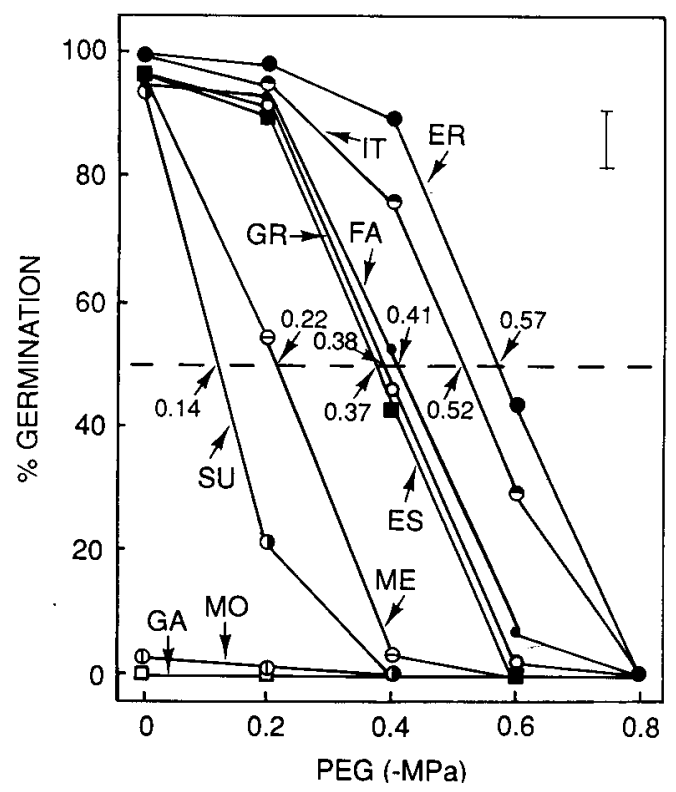

Fig. 6. Germination potential of lettuce cultivars at 30C. Seeds were soaked for $48 \mathrm{hr}$ in PEG solutions of decreasing $\psi$. The dotted line represents $50 \%$ germination. Cultivar notation as in Fig. 1. Vertical bar denotes LSD at the 0.05 probability level.

ethylene under stressful conditions. The pregermination ethylene producing capacity of the seeds, with or without a prior incorporation of ACC, was found to be much greater in slit than in nonslit seeds under stressful conditions (Table 1). However, the ethylene produced after slitting of seeds, with or without a treatment with ACC, was reduced to a greater extent by high temperature than by osmotic or salt stress.

The ability of ACC and ETH in intact and slit 'Mesa 659' seeds to improve germination potential at high temperatures also was tested. At 35C, ACC and ETH had little or no effect in inducing germination of intact seeds (zero germination potential) (Fig. 7). Untreated slit seeds had a germination potential of $0.02 \mathrm{MPa}$. Treatment with ETH or ACC increased the potential of $0.12(=0.14-0.02) \mathrm{MPa}$ and $0.14(=0.16-0.02)$ $\mathrm{MPa}$, respectively. At $32 \mathrm{C}$, intact seeds had a germination potential of $0.06 \mathrm{MPa}$. A small increase of $0.05(=0.11-0.06)$ $\mathrm{MPa}$ and large increase of 0.47 ( =0.53 - 0.06) MPa occurred due to ACC and ETH treatment, respectively. Upon slitting, the germination potential in untreated seeds increased by 0.27 $(=0.33-0.06) \mathrm{MPa}$, which can be attributed in part to the removal of the seed coat restraint (see Discussion). Unlike intact seeds, a relatively large increase of $0.17(=0.50-0.33) \mathrm{MPa}$ and a small increase of $0.07(=0.6-0.53) \mathrm{MPa}$ were induced by $\mathrm{ACC}$ and $\mathrm{ETH}$, respectively, in slit seeds.

\section{Discussion}

The results described here show a positive relationship between the ethylene producing capacity of seeds of lettuce cultivars and their ability to germinate under stressful conditions of salinity, high temperature, and osmotic restraint. Thus, ethylene production by the seed could serve as an effective biochemical marker for selecting lettuce cultivars tolerant of these stresses.

Slitting of seed coats had a varied effect on ethylene production and performance of cultivars subjected to osmotic restraint 
Table 1. Ethylene production under stressful (0.1 M NaCl, - $0.3 \mathrm{MPa}$ PEG, 35C) and nonstressful (25C) conditions in intact (I) and slit (S) 'Mesa 659' lettuce seeds with or without a prior treatment with ACC.

\begin{tabular}{|c|c|c|c|c|c|c|c|c|}
\hline \multirow[b]{3}{*}{ Treatment $^{x}$} & \multicolumn{8}{|c|}{ Ethvlene $\left(\mathrm{nl} \cdot \mathrm{hr}^{-1} \cdot \mathrm{g}^{\mathrm{l}}\right)$} \\
\hline & \multicolumn{2}{|c|}{$0.1 \mathrm{M} \mathrm{NaCl}$} & \multicolumn{2}{|c|}{$-0.3 \mathrm{MPa}$ PEG } & \multicolumn{2}{|c|}{$35 \mathrm{C}$} & \multicolumn{2}{|c|}{$25 \mathrm{C}$} \\
\hline & I & $S$ & I & $S$ & I & s & I & $\mathrm{s}$ \\
\hline Untreated & $2 \pm 1$ & $13 \pm 3$ & $3 \pm 1$ & $18 \pm 3$ & $0 \pm 0$ & $10 \pm 2$ & $9 \pm 1$ & $28 \pm 3$ \\
\hline $\mathrm{ACC}$ & $21 \pm 4$ & $144 \pm 10$ & $30 \pm 3$ & $239 \pm 17$ & $25 \pm 2$ & $64 \pm 5$ & $148 \pm 6$ & $350 \pm 21$ \\
\hline
\end{tabular}

${ }^{2}$ Data are mean \pm SE.

${ }^{x}$ Seeds were soaked for $2 \mathrm{hr}$ in water (untreated) or $10 \mathrm{~mm} \mathrm{ACC}$ at $25 \mathrm{C}$, dried, and a portion of the seeds slit; intact and slit seeds were soaked for $10 \mathrm{hr}$ in $0.1 \mathrm{M} \mathrm{NaCl}$ at 25C, $-0.3 \mathrm{MPa}$ PEG solution at 25C, and in water at 25 or $35 \mathrm{C}$.

'Seeds were rapidly wipe-dried after soaking, transferred to glass tubes, incubated for $1 \mathrm{hr}$ at $25 \mathrm{C}$, and ethylene production determined as described in Materials and Methods.

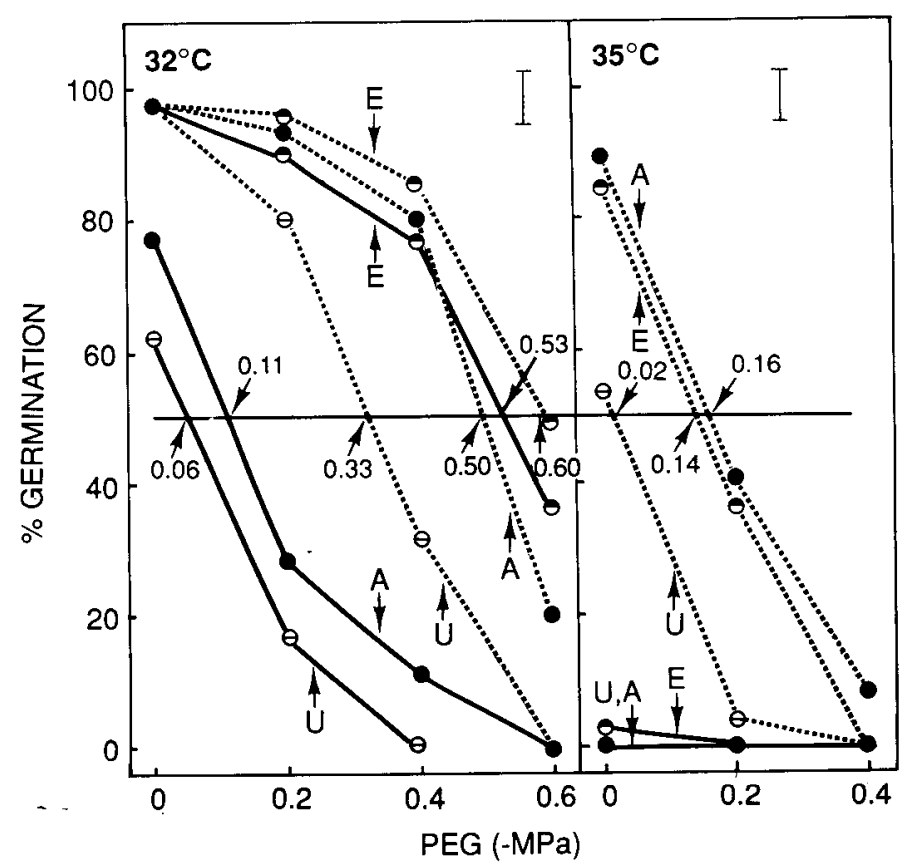

Fig. 7. The ability of intact and slit 'Mesa 659' lettuce seeds previously treated with water, ACC, or ETH to generate germination potential at 32 and $35 \mathrm{C}$. Treated and untreated seeds were soaked for $48 \mathrm{hr}$ in PEG solutions of decreasing $\psi$. Solid curves, intact seeds; dotted curves, slit seeds. Notations and concentrations: $\mathrm{U}=$ Untreated, $\mathrm{A}=10 \mathrm{mM}$ ACC, $\mathrm{E}=10 \mathrm{mM}$ ETH. The solid horizontal line represents $50 \%$ germination. Vertical bars denote LSD at the 0.05 probability level.

(Figs. 1 and 3). In some, such as 'Mesa 659' and 'Super 59', slitting greatly enhanced the ethylene production and germination. In 'Emperor', 'Fanfare', 'Empress', and 'Montello', it was moderately effective, while in 'Garnet', 'Ithaca', and 'Grand Rapids' it had no effect on germination. The data indicate that genotypic variability in seed coat characteristic may influence ethylene production and performance of seeds under stressful conditions.

The capacity to generate germination potential at the moderately high temperature of $30 \mathrm{C}$ corresponded closely with the capacity of cultivars to produce ethylene during the imposition of osmotic and salinity stresses (Figs. 1, 2, and 6), indicating that ethylene may be involved in the generation of germination potential. These data indicate that, like ethylene production, germination potential could be used as a selection criterion to identify cultivars tolerant of salt, osmotic, and high-temperature stresses.

The seed coat may reduce the performance of the growing embryo in two ways under stressful conditions: first, it may serve as a mechanical barrier and, second, it may create a hypoxic environment unfavorable for the conversion of $\mathrm{ACC}$, the immediate precursor of ethylene (Yang, 1985), to ethylene. Ethylene production increased in untreated seeds and those treated with ACC when the seed coat was slit (Table 1), indicating that the intact seed coat reduced the conversion of ACC to ethylene. It is well-known that the reaction catalyzing the conversion of ACC to ethylene by the ethylene-forming enzyme (EFE) is oxygen-dependent (Yang, 1985). The conversion of ACC to ethylene is reduced under conditions of salinity, high temperature, and hypoxia (Khan, 1989; Khan et al., 1987; Khan and Huang, 1988).

The germination potential generated by untreated, ACC-treated, and ETH-treated 'Mesa 659' seeds at 35C were insufficient to allow germination (Fig. 7). The germination potential of lettuce embryos is known to decrease as the temperature increases and, at $35 \mathrm{C}$, falls below that needed for overcoming the seed coat restraint (Takeba and Matsubara, 1979). Also, the effectiveness of ethylene in preventing thermoinhibition decreases greatly at temperatures > 32C (Abeles, 1986).

At $32 \mathrm{C}$, intact and slit seeds showed a difference in germination potential of 0.27 ( $=0.33-0.06) \mathrm{MPa}$ (Fig. 7), which may be attributed partly to the removal of the restraining force of the seed coat and partly to the potential generated by ethylene, presumably derived from the endogenous ACC (Table 1). Unlike ETH, which generated a potential of $0.47(=0.53-$ $0.06) \mathrm{MPa}, \mathrm{ACC}$ generated a potential of only $0.05(=0.11$ - 0.06) $\mathrm{MPa}$ in intact seeds. In slit seeds, the potential generated by ACC was $0.17(=0.5-0.33) \mathrm{MPa}$ and that by ETH only $0.07(=0.6-0.53) \mathrm{MPa}$. This difference indicates that slitting causes increased conversion of exogenous ACC (and perhaps also of endogenous ACC) to ethylene (Table 1), which then participates in improving the germination potential.

As the production of high levels of ethylene (Figs. 1-5), the generation of high germination potential (e.g., $0.57 \mathrm{MPa}$ for 'Emperor' vs. zero for 'Garnet' and 'Montello' at 30C) (Fig. 6) and the decreased seed coat restraint (equivalent to $0.27 \mathrm{MPa}$ ) (Fig. 7) are related to improved performance, lettuce breeders should consider developing cultivars with these traits to improve seed performance under stressful conditions. In a recent study, a preplant permeation of 'Mesa 659' lettuce seeds with ACC 
greatly improved the seedling emergence from slit seeds, but not from intact seeds planted in a soil medium at a regime of 25/35C (Khan, 1989). Evidently, the potential generated by the ACC-derived ethylene in the absence of the seed coat restraint was sufficient to induce germination and seedling emergence. Studies with rice seeds indicate that the ability to use ACC for ethylene production can be used to screen cultivars tolerant of salinity and high-temperature stress at the seedling-establishment phase (Khan, 1989; Khan et al., 1987).

Another plant hormone, cytokinin, in combination with ACC, has been shown recently to synergistically alleviate salinity and high-temperature stress on lettuce seed germination, and the alleviation of stress is accompanied by enhanced ACC use (Khan, 1989; Khan and Prusinski, 1989). A permeation of ETH and cytokinin into lettuce seeds (Sharples, 1973; Braun and Khan, 1976) in conjunction with other useful physical or chemical treatments, may be a logical approach for reducing the effect of thermoinhibition, a problem faced by lettuce growers.

\section{Literature Cited}

Abeles, F.B. 1986. Role of ethylene in Lactuca sativa $c v$. 'Grand Rapids' seed germination. Plant Physiol. 81:780-787.

Braun, J.W. and A.A. Khan. 1976. Alleviation of salinity and high temperature stress by plant growth regulators permeated into lettuce seeds via acetone. J. Amer. Soc. Hort. Sci. 101:716-721.

Ikuma, H. and K.V. Thimann. 1963. The role of seed-coats in germination of photosensitive lettuce seeds. Plant Cell Physiol. 4:169185.

Khan, A.A. 1989. Enhanced sensitivity of germination and growth processes to ethylene under stress. Proc. Intl. Congr. Plant Physiol., New Delhi. 15-20 Feb. 1988. (In press.)
Khan, A. A., M. Akbar, and D.V. Seshu. 1987. Ethylene as an indicator of salt tolerance in rice. Crop Sci. 27:1242-1247.

Khan, A.A. and X.-L. Huang. 1988. Synergistic enhancement of ethylene production and germination with kinetin and 1-aminocyclopropane-1 -carboxylic acid in lettuce seeds exposed to salinity stress. Plant Physiol. 87:847-852.

Khan, A.A. and J. Prusinski. 1989. Kinetin enhanced 1-aminocyclopropane- 1-carboxylic acid utilization during alleviation of high temperature stress in lettuce seeds. Plant Physiol. 91:733-737.

Michel, B.E. and M.R. Kaufmann. 1973. The osmotic potential of polyethylene glycol-6000. Plant Physiol. 51:914-916.

Negro, F.B. and O.E. Smith. 1978. Effect of ethylene and carbon dioxide on the germination of osmotically inhibited lettuce seeds. Plant Physiol. 62:473-476.

Rae, V. S., N. Sankhla, and A.A. Khan. 1975. Additive and synergistic effects of kinetin and ethrel on germination, thermodormancy and polyribosome formation in lettuce seeds. Plant Physiol. 56:263266 .

Saini, H. S., E.D. Consolation, P.K. Basi, and M.S. Spencer. 1986. Requirements for ethylene synthesis and action during relief of thermoinhibition of lettuce seed germination by combinations of gibberellic acid, kinetin and carbon dioxide. Plant Physiol. 81:950953.

Sharples, G.C. 1973. Stimulation of lettuce seed germination at high temperature by ethephon and kinetin. J. Amer. Soc. Hort. Sci. 98:209212.

Takeba, G. and S. Matsubara. 1979. Measurement of growth potential of the embryo in New York lettuce seed under various combinations of temperature, red light and hormones. Plant Cell Physiol. 20:5161 .

Yang, S.F. 1985. Biosynthesis and action of ethylene. HortScience 20:41-45. 